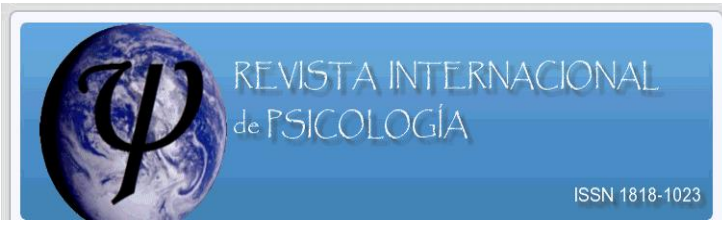

\title{
Una teoría del consumo móvil
}

\author{
Cruz García Lirios ${ }^{1}$
}

\begin{abstract}
Resumen
El comercio móvil es un indicador de la brecha económica y la consecuente, brecha digital entre los países desarrollados económicamente y los países con economías emergentes. En tal sentido, se exponen los fundamentos de una teoría para explicar las consecuencias del comercio móvil a partir de los determinantes económicos, tecnológicos, mercadotécnicos y psicológicos que llevan a una persona a adquirir productos a través de protocolos móviles.
\end{abstract}

Palabras claves; Brecha económico digital, percepción del nivel innovación, utilidad, eficiencia, adquisición y uso del servicio.

\section{Delimitación del problema}

En términos socio económico tecnológicos, la brecha digital es la extensión de la brecha económica existente entre los países desarrollados económicamente y los países con economías emergentes. Se estima que en los próximos años esta brecha digital será exponencial con el Internet móvil (observar el esquema 1 en el anexo).

En México sólo el 36\% tiene acceso telefónico, 9\% tiene una computadora personal en casa y sólo el 7\% tiene acceso a Internet. Esto implica 10 millones de dólares en ganancias cada año y un $65 \%$ de renta elevada en la telefonía móvil si se compara con las tarifas internacionales.

En contraste, los países con economías emergentes más exitosas (Taiwan y Hong Kong) han acortado ambas brechas: la económica y la digital.

En el mundo, el sector de telecomunicaciones (Televisión, Internet y telefonía) crece un $25 \%$ anual. Es un mercado privatizado, el $98 \%$ de las empresas que ofrecen el servicio son controladas por AOL, AT\&T, T-mobile a través de filiales o alianzas estratégicas. Durante la década de los ochenta y noventa, Internet fue el subsector que más creció con un $20 \%$ al año. Al incorporarse este servicio al de telefonía móvil el número de usuarios y clientes potenciales crecen un $15 \%$ anual.

Sin embargo, en términos socio psicológicos, los países con economías emergentes tienden a consumir informalmente tanto productos como servicios. En la Ciudad de México, el comercio informal representa el $40 \%$ de las ventas y servicios en las zonas periféricas y marginadas. Consecuentemente, las personas tienden a percibir el producto antes de comprarlo en tianguis, bazares o autoservicios. Esta sería la causa principal por la cual quienes tienen acceso a Internet no compran productos telefónicos móviles. Sin embargo,

1 Universidad Autónoma Metropolitana. Correo electrónico: csh96327267@titlani.uam.mx 
los consumidores perciben la utilidad de un producto o servicio más a partir de los costos sospechados que de los beneficios ofrecidos.

Consecuentemente, en términos socio psico ambientales, se generan 50 millones de toneladas de desechos industriales y tecnológicos por la producción y consumo de las innovaciones telefónicas siendo Motorola la empresa con menos estándares de calidad al fabricar productos con un $19 \%$ de cobre y $8 \%$ de acero (principales agentes de toxicidad).

En este sentido, el consumo móvil podría orientarse a la adquisición de productos y servicios científicos considerando que las fuentes de información en Internet han crecido paulatina y establemente (Observar el esquema 2 en el anexo).

Por lo tanto, una teoría que explique las consecuencias del comercio móvil, será fundamental en la elaboración de programas sustentables.

Hasta aquí los fundamentos socio históricos de la teoría del consumo móvil. A continuación los fundamentos teóricos.

\section{Conceptuación del problema}

Son siete las aproximaciones antecedentes de la teoría del consumo móvil.

Teoría Universal de Valores. Analiza las necesidades personales, establece una jerarquía de principios y prioridades derivadas de un grupo de referencia (Schwartz, 1992).

Teoría de la Motivación Humana. Sostiene que las necesidades al estar jerarquizadas fundamentan las razones para llevar a cabo una acción. Esto es, activan procesos deliberados que llevan a las personas a sistematizar sus objetivos (Maslow, 1943).

Teoría de la Acción Razonada. Delimita el efecto indirecto de las creencias generales sobre el comportamiento deliberado. Dicho efecto es transmitido por actitudes, percepciones, normas e intenciones. Estas trayectorias al ser deliberadas y sistemáticas, implican un cambio al interior del individuo más que del grupo (Ajzen \& Fishbein, 1974).

Teoría de la Conducta Planificada. Análogamente, las creencias determinan indirectamente un comportamiento delimitado, deliberado, planificado, y consecuentemente, sistemático a través de actitudes, percepciones, normas e intenciones en el individuo (Ajzen, 1991).

Teoría de la Aceptación de la Tecnología. Define las percepciones específicas en torno a la utilidad y la facilidad de uso de alguna tecnología, las que predicen directamente a las intenciones de llevar a cabo comportamientos auxiliados por dicha tecnología (Davis, 1989).

Teoría del Comercio Electrónico. Así mismo, estas mismas percepciones utilitarias y las percepciones de riesgos influyen indirectamente sobre comportamientos de compra en Internet a través de habilidades e intenciones de compra (Lee, Park \& Ahn, 2000). 
Teoría Social Cognitiva. Insiste en la interacción entre las habilidades individuales y los desafíos que le marca el entorno cuyos resultados son la adaptación de la conducta o la transformación del medio ambiente (Bandura, 1989).

Las teorías de valores y motivacionales explicaron las necesidades que llevan a los consumidores a adquirir determinados productos. Consecutivamente, la teoría de acción razonada explicó el consumo en centros comerciales. Posteriormente, la teoría de la conducta planificada explicó el consumo en autoservicios. Sucesivamente, la teoría de la aceptación de la tecnología explicó el consumo de computadoras personales y telefonía móvil. Consecuentemente, la teoría de la adopción del comercio explicó los riesgos que impiden una transacción digital. Finalmente, la teoría social cognitiva explicó la interacción entre el usuario y la red.

En cada aproximación se han desarrollado modelos en los que se incluyen variables latentes. Precisamente, la teoría del consumo móvil explica:

El grado de innovación tecnológica que influirá indirectamente (a través de las percepciones utilitarias, la identificación promocional y la auto eficiencia móvil) sobre la adquisición de productos y servicios en el Internet móvil.

Hasta aquí los fundamentos teóricos de la teoría del consumo móvil. A continuación se exponen sus fundamentos metodológicos.

\section{Modelación del problema}

La estructura de un modelo se establece a partir de la formula: Parámetros totales de un modelo = parámetros de varianzas y covarianzas de los constructos exógenos + parámetros de varianzas y covarianzas de las perturbaciones de las trayectorias + parámetros de efectos directos e indirectos sobre los constructos endógenos (Kline, 1998).

Un modelo es un plan sistemático en el que se establece la verosimilitud de las relaciones causales entre las variables que explican un problema. En dicho modelo se incluyen dos tipos de variables: manifiestas y a partir de ellas, se infieren variables latentes mediante un análisis factorial (observar el esquema 3 en el anexo).

En ambos tipos de análisis, la carga factorial (la relación entre la variable latente con las variables manifiestas) determina la inclusión de una variable manifiesta en una variable latente. Sin embargo, sólo en el análisis confirmatorio se plantean hipótesis en torno a las relaciones entre las variables manifiestas y la variable latente (Pedhazur \& Schmelkin, 1991).

Ambos tipos de análisis factorial establecen la validez convergente de la variable latente.

Ahora bien, ambas variables, manifiesta y latente pueden ser modeladas como exógenas y endógenas, moderadoras y mediadoras. Serán exógenas cuando determinen a otra variable, endógenas cuando sean predecidas por otra variable, moderadoras cuando interactuen con 
otra variable y mediadora cuando transmitan los efectos de una variable exógena sobre una endógena (Kline, 2006).

Un proceso moderador es definido como la fragmentación de los efectos directos de un grupo de dos o más entidades independientes sobre una entidad focal dependiente. Es decir, el efecto de una entidad independiente sobre una entidad focal dependiente disminuye o aumenta en función de la relación de una tercera entidad independiente con la primera entidad independiente. Dicho proceso moderador nos permite establecer cuándo ocurrirán los efectos expuestos (Baron \& Kenny, 1986).

En el proceso moderador están incluidas tres condicionantes:

La entidad A debe estar directa y significativamente relacionada con la entidad C.

La entidad A no debe estar alta y significativamente relacionada con la entidad B.

La entidad B debe estar directa y significativamente relacionada con la entidad C.

En tanto, un proceso mediador es definido como la transmisión de los efectos de un grupo de entidades independientes sobre una entidad focal dependiente. Dicho proceso mediador devela cómo y por qué ocurren los efectos expuestos.

En el proceso mediador están incluidas tres condicionantes:

Las entidades A y B no deben estar directa y significativamente relacionadas con la entidad C.

La entidad D debe estar directa y significativamente relacionada con la entidad C

Las entidades A y B deben estar alta y significativamente relacionados con la entidad D.

Cabe señalar que los procesos moderadores son más frecuentes que los procesos mediadores. Por consiguiente, ambos procesos moderadores y mediadores se incluyen en una notación estructural.

Considerando estas recomendaciones, se esquematizó un modelo en torno al consumo móvil una vez planteada la pertinencia de variables a partir de los avances y limitaciones (validez de reportes verbales, pertinencia de constructos e instrumentos, captación de homogeneidad y diferenciación en los niveles de análisis) en los estudios de percepciones, creencias, actitudes, motivos, habilidades, intenciones y comportamientos derivados del uso de las tecnologías de información y comunicación (observar el esquema 4 en el anexo).

La modelación de las variables planteadas en fases consecutivas que van desde lo situacional hasta lo conductual explican qué lleva a las personas a realizar ciertas practicas y a sistematizarlas, está representada por las relaciones causales directas de constructos exógenos sobre endógenos e indirectas con constructos mediadores,

La percepción de utilidad es una variable latente que explica un proceso de adquisición de un producto o servicio en Internet. Esto implica tres cuestiones:

Al ser una variable latente, la percepción de utilidad incluye variables manifiestas que han configurado modelos explicativos del consumo deliberado, planificado y sistemático. 
Dichos modelos explicativos se construyeron a partir de teorías que se modificaron en función de los hallazgos y su poder predictivo.

Consecuentemente, la teoría del consumo móvil es la evolución de las aproximaciones valorativas, motivacionales, razonadas, planificadas, de aceptación tecnológica y cognitiva social.

Así mismo, el modelo del consumo móvil representa la optimización de modelos antecedentes tales como; acción razonada, conducta planificada, aceptación de la tecnología y adopción del comercio electrónico.

De este modo, la variable latente de la utilidad percibida es la continuación avanzada de variables manifiestas tales como; la percepción de riesgo, los valores de consumo, las actitudes hacia los productos o servicios y los motivos de compra.

Por lo tanto, la percepción de utilidad puede ser incluida como variable exógena y puede relacionarse causalmente con otras variables como lo plantea el modelo del consumo móvil.

\section{Evaluación del problema}

¿Cómo son las relaciones entre los constructos que explican el impacto de las innovaciones tecnológicas en el consumo?

\subsection{Sujetos}

Se seleccionarán 400 residentes de la ZMVM; 300 en la confiabilidad y validación de los instrumentos que medirán las cinco variables y 100 en la prueba de ajuste del modelo.

\subsection{Variables}

La percepción del grado de innovación. Son las funciones y características de un producto y/o servicio percibidas por los usuarios.

La percepción del nivel de utilidad. Es la expectativa evaluativa, actitudinal y motivacional de mayores beneficios y menores costos en torno al consumo de un producto o servicio.

La percepción del grado de eficiencia. Es el manejo de un producto y/o servicio para fines de consumo.

La propensión al consumo. Es la tendencia a comprar un producto y/o servicio.

El nivel de uso. Es el tiempo de compra de un producto o adquisición de un servicio.

\subsection{Hipótesis}

La percepción del grado reinnovación tiene un efecto indirecto, positivo y significativo sobre el nivel de uso. Dicho efecto, esta mediado por la percepción del nivel de utilidad, del grado de eficiencia y de la propensión al consumo. 


\subsection{Objetivos}

En la primera fase, se construirán y se establecerá la confiabilidad y validez de los instrumentos que medirán las cinco variables.

En la segunda fase, se modelarán y se demostrará la verosimilitud del ajuste de las relaciones causales indirectas y directas, negativas y positivas, y significativas entre las cinco variables mediante análisis de ecuaciones estructurales.

\subsection{Instrumentos}

Los instrumentos (observar el cuestionario en el anexo) fueron construidos con base en las tres fases siguientes.

Conceptuación de la variable que se quiere medir.

Definición y distinción de otras variables.

Generalidad y especificación en el contenido contextual.

Coherencia en la selección de indicadores.

Elaboración de un número de reactivos proporcional tres o cuatro veces más a la escala final.

Evitación de conectivos excesivos en el reactivo.

Combinación de reactivos con un sentido positivo y negativo.

Determinación del formato de medición.

Selección de reactivos que reflejan el propósito de la escala. Procuración de que a cada reactivo mide un rasgo o atributo de la variable.

Definición del tipo de escala: absoluta en la que el sujeto indica su preferencia ante un solo estímulo o comparativas en la que se le pide al sujeto que indique su preferencia ante muchos estímulos.

Especificación de las opciones de respuestas.

Indicación del tiempo aproximado de respuesta.

Homogeneización y detección de sesgos a través de los jueces en cuanto a experiencia, estructuración y características individuales.

Piloteo de reactivos para desarrollo de una muestra.

Revisión inicial de las respuestas a los reactivos.

Asignación de un número a un atributo de un objeto a partir de condicionantes.

Codificación reversa de los reactivos con un sentido negativo.

Optimización de la longitud de la escala.

Supresión de los reactivos contestados con una sola opción de respuesta y aquellos en los que se contestaron pocos reactivos.

Inclusión de reactivos confiabilizados y validados en el instrumento final. 


\subsection{Procedimiento}

La confiabilidad y validez de los instrumentos que miden las variables del modelo se realizará con 300 residentes elegidos de forma aleatoria.

La verosimilitud del ajuste del modelo a los datos reales, se realizará con 100 residentes seleccionados de un modo aleatorio.

Hasta aquí los fundamentos metodológicos de la teoría del consumo móvil. A continuación se exponen sus fundamentos estadísticos.

\section{Análisis del problema}

Se calculará su confiabilidad mediante la técnica estadística de alfa de Cronbach. Mayor variabilidad de los datos equivale a una mayor confiabilidad.

Se realizará una transformación monotómica que consistirá en sumar o multiplicar una constante sin alterar la distancia entre una variable y otra para estandarizarlas y se seleccionará a aquellos reactivos que tendrán una distribución de kurtosis entre - .5 y .5 para evitar que sus datos fuesen negativos e inferiores a la media o bien, para evitar que sus datos fuesen positivos y superiores a la media. También se seleccionará a aquellos reactivos con un sesgo entre -.3 y .3

Se validarán convergentemente los constructos a partir de la correlación entre los reactivos con la variable latente y se establecerá su validez divergente mediante la escasa correlación con otras variables latentes.

Se estimarán coeficientes de correlación (r) entre cada variable, se identificará el grado de asociación indicado por los valores altos o bajos de una variable que se relacionarán con los valores altos o bajos de otra variable.

Se estimará el coeficiente de correlación múltiple (R) el cual indicará la medida del grado de exactitud de la predicción (efectos exclusivos del predictor sobre el criterio) oscilando entre -1 hasta +1

Así mismo, el coeficiente de regresión $(B / \beta)$ el cual indicará la influencia que cada predictor tendrá sobre el criterio. En tal sentido, el análisis de trayectorias se utilizará para establecer las relaciones causales, moderadoras, mediadoras y no causales entre variables y errores de medición.

Se elaborará un modelo de medición y un modelo estructural. El modelo de medición planteará hipotéticamente las relaciones causales y no causales entre los constructos exógenos y mediadores, dichas relaciones se orientarán a la predicción de un constructo endógeno. Se calculará la varianza explicada, se establecerán los errores de estimación. Dicho procedimiento se planteará en matrices de ecuaciones. 
De este modo, los indicadores de un constructo tendrán un aceptable ajuste sobre un factor del modelo siendo los grados de libertad los que identificarán los constructos evaluados por su ajuste.

La evaluación del ajuste se establecerá mediante estimadores tales como; Índice de Ajuste Comparativo (Comparative Fit Index CFI), Índice de ajuste Normado (Normed Fit Index NFI) e Índice de Bondad de Ajuste (Goodness of Fit Index GFI). Sin embargo, dichos parámetros sólo indicarán el ajuste global del modelo pero no su significancia. Además un buen ajuste no indicará la parsimonia del modelo, sólo se analizarán las varianzas de las perturbaciones que pueden indicar una relación baja entre variables exógenas y endógenas.

Hasta aquí los fundamentos estadísticos de la teoría del consumo móvil. A continuación se exponen sus aplicaciones.

\section{Discusión del problema}

En una economía regulada por la competencia en el mercado se esperará que el cliente potencial desarrolle creencias, habilidades y conocimientos de consumo. Se esperará que estas competencias (satisfacción de necesidades) aparezcan principalmente en un mercado móvil de consumo. Es decir, las personas desde un teléfono celular comprarán y venderán productos y servicios buscando la mejor oferta y sus correspondientes beneficios. En consecuencia, la explicación de tal proceso improvisado y asistemático, necesariamente alude a variables de índole afectivas más que cognitivas como el modelo del consumo móvil plantea. Es así como la identidad hacia un producto, servicio o marca será una variable relevante en la investigación en torno al comercio móvil.

A partir de un análisis de spots se establecerán los valores agregados de los productos y servicios móviles. En efecto, el móvil es más un valor agregado de un producto en el que se busca activar emociones más que la reflexión sobre el deterioro ambiental. De este modo, un producto que en su proceso de producción y consumo impide el desarrollo sostenido, es promocionado como una alternativa de solución ante dicho problema. En tal sentido, los valores agregados de los productos y los servicios son determinantes en las tomas de decisión. A partir de este análisis se establecerán las condiciones necesarias para la toma de dicha decisión. Esto implica; mensajes activadores (promteos) en recipientes cercanos a personas que utilizan el móvil en exceso, información visual, guías de reciclaje y reutilización, así como información personalizada. 


\section{Referencias}

Ajzen, I. (1991). The Theory of Planned Behavior. Organizational Behavior and Human Decision Processes. 50, 179-211

Ajzen, I. \& Fishbein, M. (1974). Factors influencing intentions and the intention behavior relation. Human Relations. 27, 1-15

Bandura, A. (1989). Human agency in Social Cognitive Theory. American Psychologist. $44,1175-1184$

Baron, R. \& Kenny, D. (1986). The moderator and mediator variables distinction in social psychology research. Conceptual strategic, and statistical considerations. Journal of Personality and Social Psychology. 51, 1173-1182

Davis, F. D. (1989). Perceived usefulness, perceived ease of use and user acceptance of information technology. Management Information System Quarterly 13, 319-340

Kline, R. (1998). Principles and practice of structural equations modelling. New York: Guilford Press.

Kline, R. (2006). Reverse arrow dynamics. Formative measurement and feedback loops. In G. Hancock \& R. Mueller (Eds.). Structural Equation Modeling: A Second Course. (pp. 43-68). Greenwood, CT: Information Age Publishing, Inc.

Lee, D., Park, J. \& Ahn, J. (2000). On the explanation factors affecting e-commerce adoption. Working Paper. 1-21

Maslow, A. H. (1943). A theory of human motivation. Psychological Review. 50, 370-396

Pedhazur, E. \& Schmelkin, L. (1991). Measurement, Design, and Analysis: An Integrated Approach. Hillsdale, New Jersey, Lawrence Erlbaum Associates, Publishers.

Schwartz, S. (1992). Universal in the content and structure of values: theoretical advances and empirical test in 20 countries. Advances in Experimental Social Psychology. 25, $1-65$ 


\section{Anexos}

\section{Esquemas}

Esquema 1. Situación anual de las telecomunicaciones

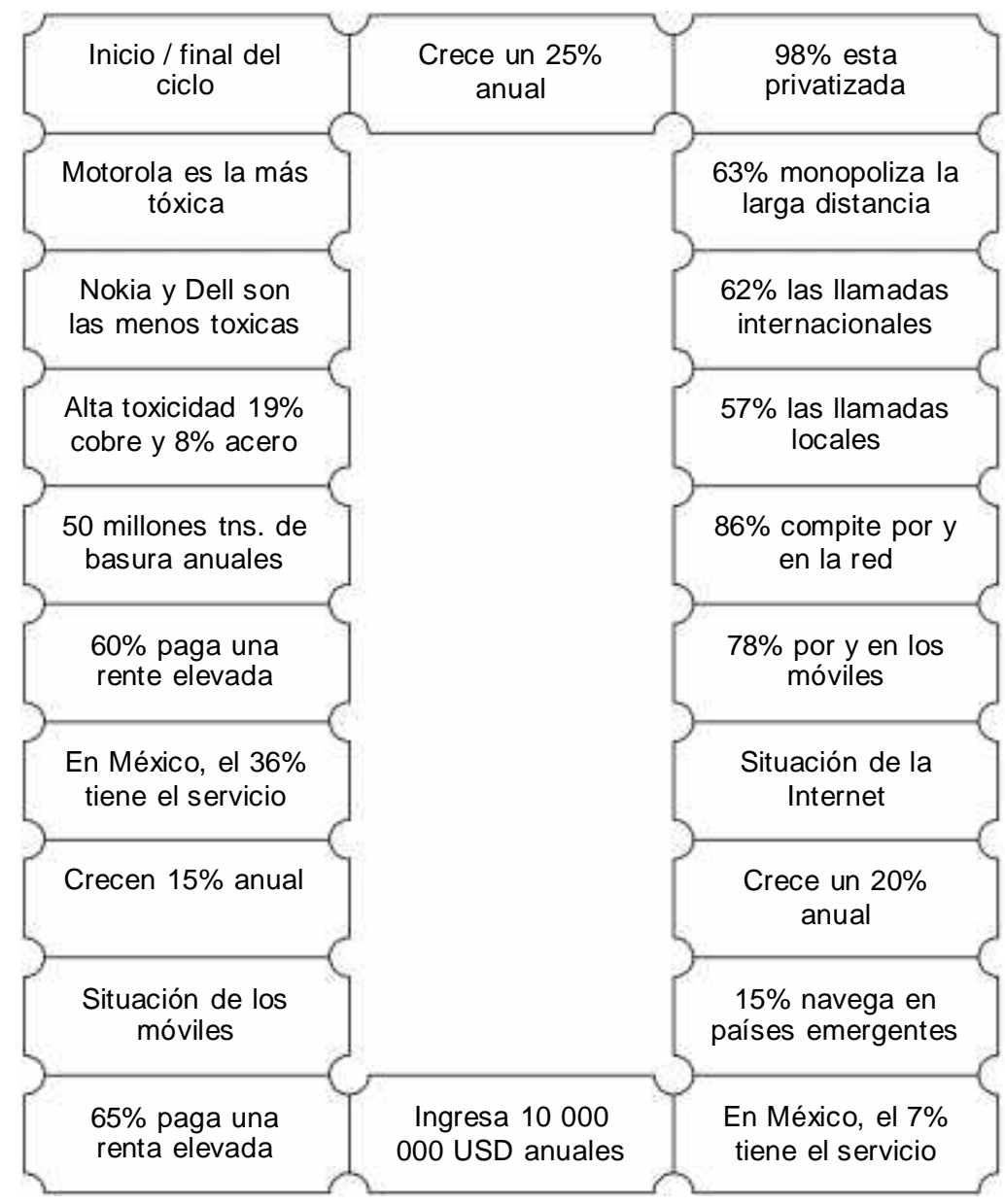

Fuente: Elaborado con base en las estadísticas de las telecomunicaciones mundiales y Greenpace (2006) 
Esquema 2. Situación anual de las revistas científicas electrónicas en Ibero América

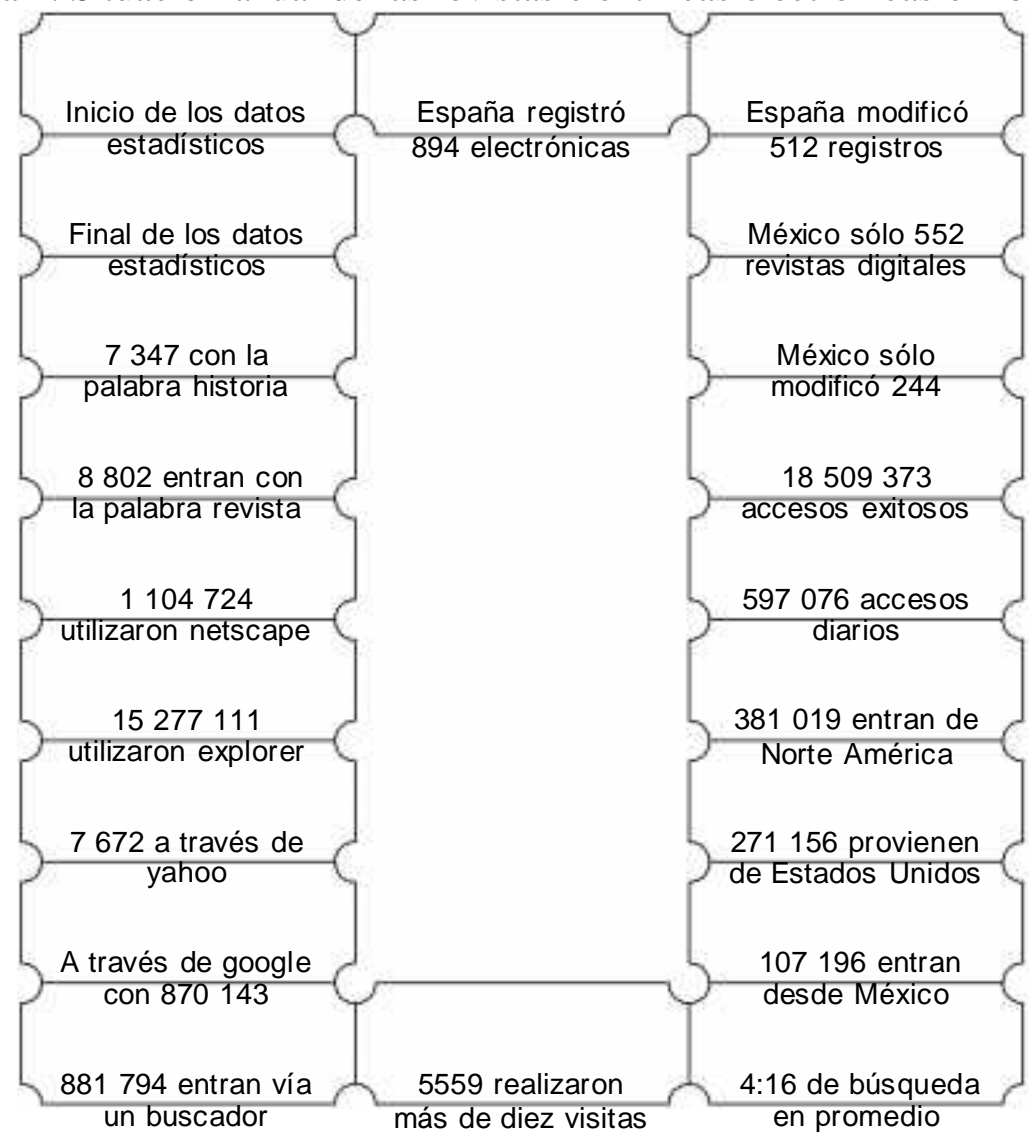

Fuente: Elaborado con base en las estadísticas de Latindex (2006); Scielo (2006) 
Esquema 3. Notación estructural

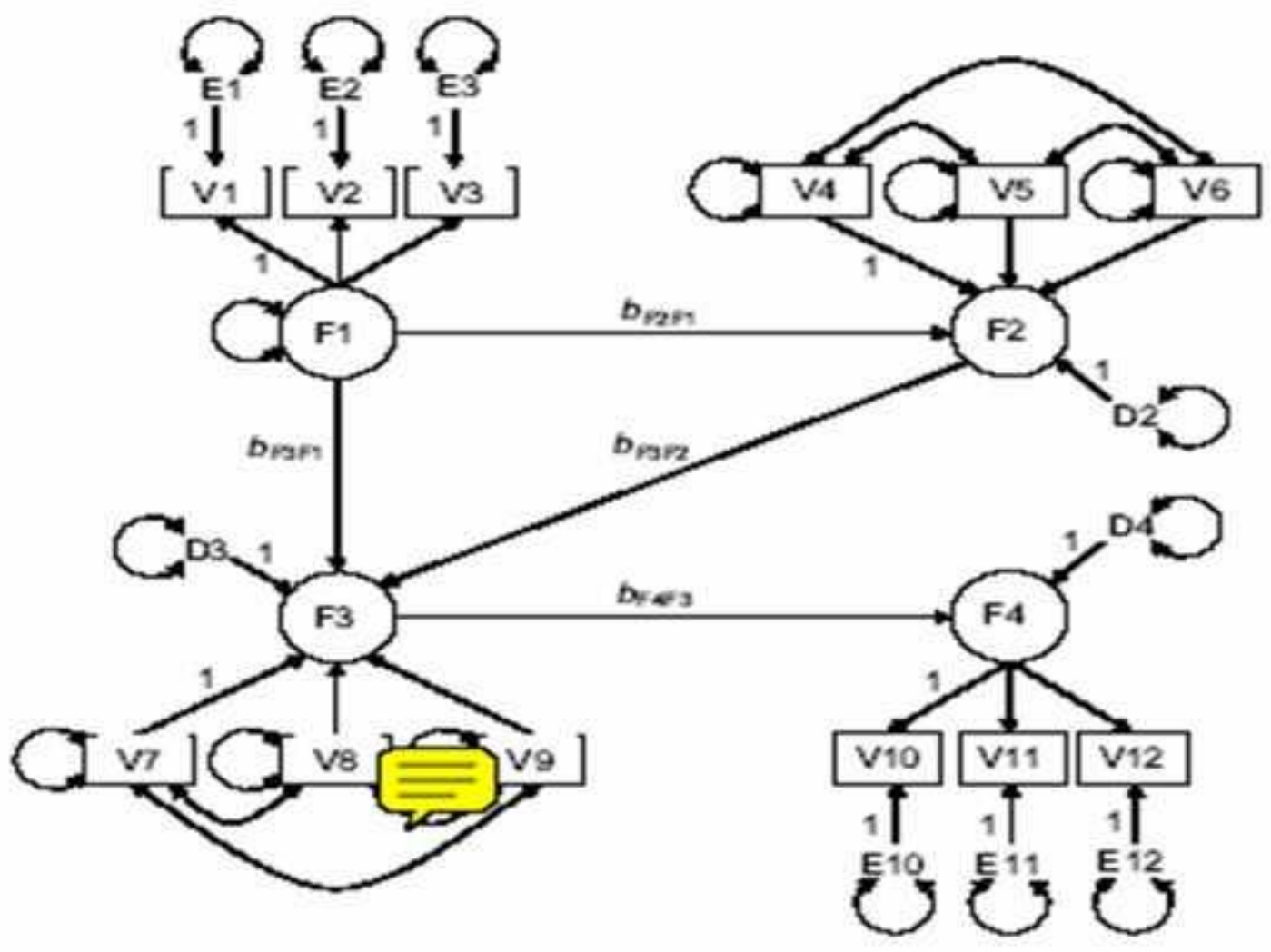

Fuente: Kline (2006) 


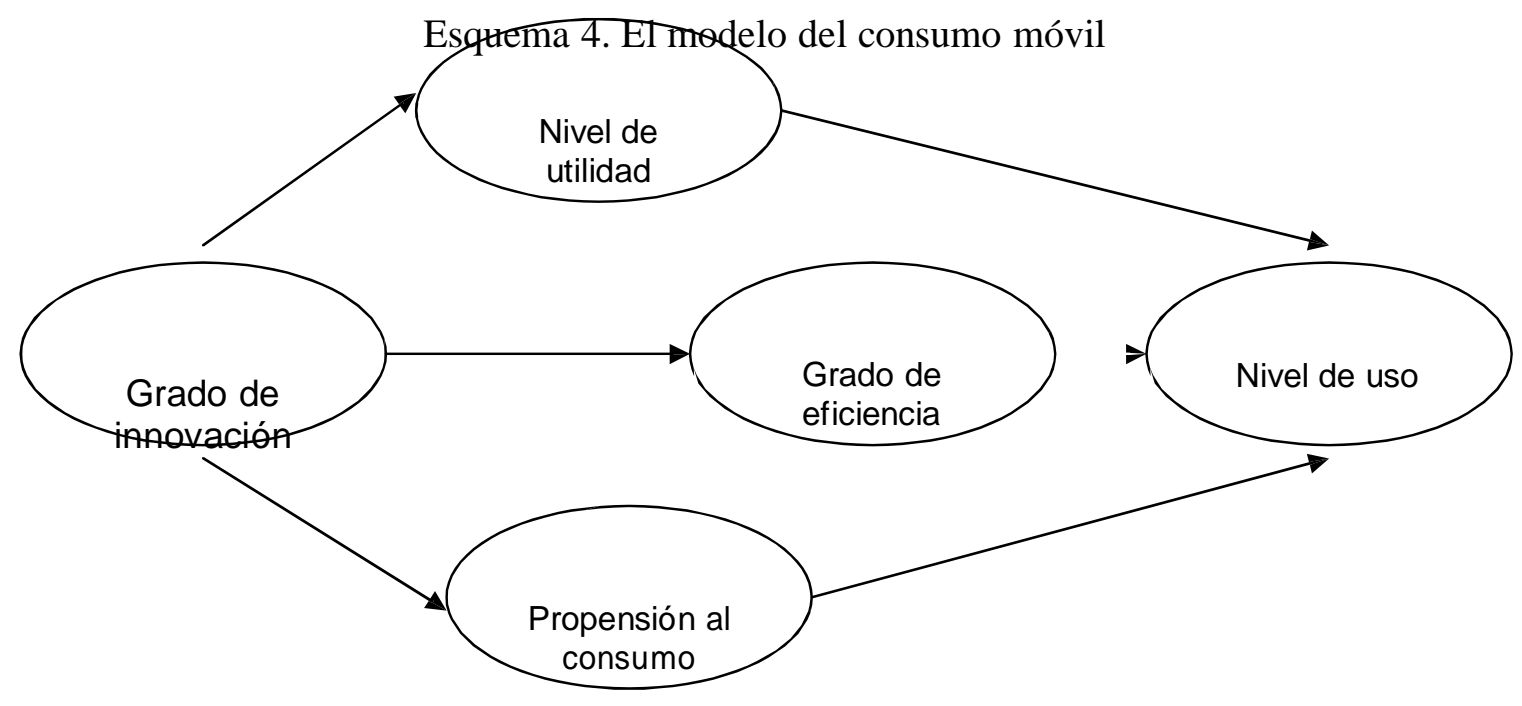

Fuente: Elaboración propia con base en el estado del arte 


\section{Cuestionario}

Número de cuestionario

Apreciable estudiante:

La licenciatura de psicología social de la Universidad Autónoma Metropolitana, unidad Iztapalapa esta realizando una encuesta sobre el servicio de telefonía móvil.

Tacha con una x en la opción que corresponda.

Sexo: Masculino ( ) Femenino ( )

Ingresos mensuales: menos de 3000 ( ) entre 3000 y 6000 ( ) mas de $6000($ )

División: CBI ( ) CBS ( ) CSH ( )

Grado: Licenciatura ( ) Maestría ( ) Doctorado ( )

Ahora, por favor revisa la marca de tu teléfono y la compañía que te otorga el servicio y tacha con una $\mathrm{x}$ en la opción correspondiente.

Marcas: Nokia ( ) Motorola ( ) LG ( ) Ericson ( ) Samsung ( )

Compañías: Telcel ( ) Nextel ( ) Iusacel ( ) Unefon ( ) Movistar ( )

Plan: tarifarlo ( ) renta ( )

Costo al mes: menos de 3000 ( ) entre 3000 y 6000 ( ) mas de 6000 ( )

\section{Ahora marca con una $x$ en el paréntesis de cada reactivo.}

Un celular con identificador de llamadas es:

Muy obsoleto ( ) obsoleto ( ) actualizado ( ) muy actualizado ( )

Un celular con manos libres es:

Muy obsoleto ( ) obsoleto ( ) actualizado ( ) muy actualizado ( )

Un celular con tiempo aire es:

Muy obsoleto ( ) obsoleto ( ) actualizado ( ) muy actualizado ( )

Un celular con fotos es:

Muy obsoleto ( ) obsoleto ( ) actualizado ( ) muy actualizado ( )

Un celular que recibe llamadas en el metro es: 
Muy obsoleto ( ) obsoleto ( ) actualizado ( ) muy actualizado ( )

Un celular con video juegos es:

Muy obsoleto ( ) obsoleto ( ) actualizado ( ) muy actualizado ( )

Un celular con cámara de video es:

Muy obsoleto ( ) obsoleto ( ) actualizado ( ) muy actualizado ( )

Un celular con antena es:

Muy obsoleto ( ) obsoleto ( ) actualizado ( ) muy actualizado ( )

Un celular con mp3 es:

Muy obsoleto ( ) obsoleto ( ) actualizado ( ) muy actualizado ( )

Un celular con canales de televisión es:

Muy obsoleto ( ) obsoleto ( ) actualizado ( ) muy actualizado ( )

Un celular con cobertura nacional es:

Muy obsoleto ( ) obsoleto ( ) actualizado ( ) muy actualizado ( )

Un celular analógico es:

Muy obsoleto ( ) obsoleto ( ) actualizado ( ) muy actualizado ( )

Un celular con chat es:

Muy obsoleto ( ) obsoleto ( ) actualizado ( ) muy actualizado ( )

Un celular msn es:

Muy obsoleto ( ) obsoleto ( ) actualizado ( ) muy actualizado ( )

Un celular con audífonos es:

Muy obsoleto ( ) obsoleto ( ) actualizado ( ) muy actualizado ( )

Un celular con AM/FM es:

Muy obsoleto ( ) obsoleto ( ) actualizado ( ) muy actualizado ( )

Un celular con correo de voz es:

Muy obsoleto ( ) obsoleto ( ) actualizado ( ) muy actualizado ( )

Un celular con mensajes escritos es: 
Muy obsoleto ( ) obsoleto ( ) actualizado ( ) muy actualizado ( )

\section{Ahora marca con una $x$ en el paréntesis de cada reactivo.}

Con este servicio compraré las cosas que busco.

Muy en desacuerdo ( ) en desacuerdo ( ) de acuerdo ( ) Muy de acuerdo ( )

Con este servicio chatearé con el tipo de personas que busco.

Muy en desacuerdo ( ) en desacuerdo ( ) de acuerdo ( ) Muyde acuerdo ( )

Con este servicio consultaré los artículos científicos que necesito.

Muy en desacuerdo ( ) en desacuerdo ( ) de acuerdo ( ) Muy de acuerdo ( )

Con este servicio contrataré otros servicios que busco.

Muy en desacuerdo ( ) en desacuerdo ( ) de acuerdo ( ) Muy de acuerdo ( )

Con este servicio chatearé con personas tímidas.

Muy en desacuerdo ( ) en desacuerdo ( ) de acuerdo ( ) Muy de acuerdo ( )

Con este servicio consultaré la información académica que necesito.

Muy en desacuerdo ( ) en desacuerdo ( ) de acuerdo ( ) Muy de acuerdo ( )

Con este servicio mejoraré mis criterios de compra.

Muy en desacuerdo ( ) en desacuerdo ( ) de acuerdo ( ) Muy de acuerdo ( )

Con este servicio desarrollaré mis habilidades comunicativas.

Muy en desacuerdo ( ) en desacuerdo ( ) de acuerdo ( ) Muy de acuerdo ( )

Con este servicio aprenderé a encontrar cualquier tipo de información.

Muy en desacuerdo ( ) en desacuerdo ( ) de acuerdo ( ) Muy de acuerdo ( )

Con este servicio fácilmente compras lo que te gusta.

Muy en desacuerdo ( ) en desacuerdo ( ) de acuerdo ( ) Muy de acuerdo ( )

Con este servicio fácilmente contratas otros servicios que te gustan.

Muy en desacuerdo ( ) en desacuerdo ( ) de acuerdo ( ) Muy de acuerdo ( )

Con este servicio fácilmente te inscribes a los cursos que necesites. 
Muy en desacuerdo ( ) en desacuerdo ( ) de acuerdo ( ) Muy de acuerdo ( )

\section{Ahora marca con una $x$ en el paréntesis de cada reactivo.}

Con este servicio puedo eliminar las ofertas inesperadas.

Nunca ( ) casi nunca ( ) casi siempre ( ) siempre ( )

Con este servicio puedo evitar la publicidad erótica repentina.

Nunca ( ) casi nunca ( ) casi siempre ( ) siempre ( )

Con este servicio puedo elegir la información científica que necesito.

Nunca ( ) casi nunca ( ) casi siempre ( ) $\quad$ siempre ( )

Con este servicio puedo seleccionar las ofertas que necesito.

Nunca ( ) casi nunca ( ) casi siempre ( ) siempre ( )

Con este servicio puedo elegir con quien chatear.

Nunca ( ) casi nunca ( ) casi siempre ( ) $\quad$ siempre ( )

Con este servicio puedo inscribirme a los cursos que necesito.

Nunca ( ) casi nunca ( ) casi siempre ( ) $\quad$ siempre ( )

Con este servicio puedo comprar los productos que busco.

Nunca ( ) casi nunca ( ) $\quad$ casi siempre ( ) $\quad$ siempre ( )

Con este servicio puedo contratar otros servicios que me gusten.

Nunca ( ) casi nunca ( ) casi siempre ( ) $\quad$ siempre ( )

Con este servicio puedo chatear con las comunidades científicas que busco.

Nunca ( ) casi nunca ( ) casi siempre ( ) $\quad$ siempre ( )

Con este servicio puedo encontrar ofertas especiales.

Nunca ( ) casi nunca ( ) casi siempre ( ) $\quad$ siempre ( )

Con este servicio puedo bajar imágenes eróticas especiales.

Nunca ( ) casi nunca ( ) casi siempre ( ) $\quad$ siempre ( ) 
Con este servicio puedo comunicarme con los científicos que busco.

Nunca ( )

$$
\text { casi nunca ( ) }
$$

Con este servicio compraría los productos en oferta.

Muy improbable ( ) imposible ( ) posible ( ) muyprobable ( )

Con este servicio contrataría los servicios en oferta.

Muy improbable ( ) imposible ( ) posible ( ) muy probable ( )

Con este servicio adquiriría la información en oferta.

Muy improbable ( ) imposible ( ) posible ( ) muy probable ( )

Con este servicio compraría productos piratas.

Muy improbable ( ) imposible ( ) posible ( ) muyprobable ( )

Con este servicio contrataría servicios clandestinos.

Muy improbable ( ) imposible ( ) posible ( ) muy probable ( )

Con este servicio adquiriría información esotérica.

Muy improbable ( ) imposible ( ) posible ( ) muy probable ( )

Con este servicio compraría productos de prestigio.

Muy improbable ( ) imposible ( ) posible ( ) muy probable ( )

Con este servicio contrataría servicios de calidad.

Muy improbable ( ) imposible ( ) posible ( ) muy probable ( )

Con este servicio adquiriría información relevante.

Muy improbable ( ) imposible ( ) posible ( ) muyprobable ( )

Con este servicio compraría productos de moda.

Muy improbable ( ) imposible ( ) posible ( ) muyprobable ( )

Con este servicio contrataría otros servicios actualizados.

Muy improbable ( ) imposible ( ) posible ( ) muy probable ( )

Con este servicio adquiriría información de frontera.

Muy improbable ( ) imposible ( ) posible ( ) muy probable ( ) 


\section{Ahora marca con una $x$ en el paréntesis de cada reactivo}

Con este servicio compras un video en:

Menos de 10 minutos ( ) entre 10 y $15($ ) entre 15 y $20($ ) mas de $20($ )

Con este servicio contratas un servicio erótico en:

Menos de 10 minutos ( ) entre 10 y 15 ( ) entre 15 y 20 ( ) mas de 20 ( )

Con este servicio adquieres un artículo científico en:

Menos de 10 minutos ( ) entre 10 y 15 ( ) entre 15 y $20($ ) mas de 20 ( )

Con este servicio compras una melodía en:

Menos de 10 minutos ( ) entre 10 y $15($ ) entre 15 y $20($ ) mas de $20($ )

Con este servicio contratas otros servicios de telefonía en:

Menos de 10 minutos ( ) entre 10 y $15($ ) entre 15 y $20($ ) mas de $20($ )

Con este servicio adquieres un libro en:

Menos de 10 minutos ( ) entre 10 y $15($ ) entre 15 y $20($ ) mas de $20($ )

Con este servicio compras boletos para un concierto en:

Menos de 10 minutos ( ) entre 10 y 15 ( ) entre 15 y 20 ( ) mas de 20 ( )

Con este servicio contratas un servicio de mensajería en:

Menos de 10 minutos ( ) entre 10 y 15 ( ) entre 15 y 20 ( ) mas de 20 ( )

Con este servicio adquieres una computadora en:

Menos de 10 minutos ( ) entre 10 y $15($ ) entre 15 y $20($ ) mas de $20($ )

Con este servicio compras boletos de avión en:

Menos de 10 minutos ( ) entre 10 y $15($ ) entre 15 y $20($ ) mas de $20($ )

Con este servicio contratas un servicio bancario en:

Menos de 10 minutos ( ) entre 10 y $15($ ) entre 15 y $20($ ) mas de $20($ )

Con este servicio te inscribes a cursos en:

Menos de 10 minutos ( ) entre 10 y 15 ( ) entre 15 y 20 ( ) mas de 20 ( ) 


\section{(c) (1)}

Este texto está protegido por una licencia Creative Commons

$$
\text { 4.0. }
$$

Usted es libre para Compartir — copiar y redistribuir el material en cualquier medio o formato - y Adaptar el documen- to -remezclar, transformar y crear a partir del material- para cualquier propósito, incluso comercialmente, siempre que cumpla la condición de:

Atribución: Usted debe reconocer el crédito de una obra de manera adecuada, proporcionar un enlace a la licencia, e in- dicar si se han realizado cambios. Puede hacerlo en cualquier forma razonable, pero no de forma tal que sugiera que tie- ne el apoyo del licenciante o lo recibe por el uso que hace.

$\underline{\text { Resumendelicencia - Textocompletodelalicencia }}$ 